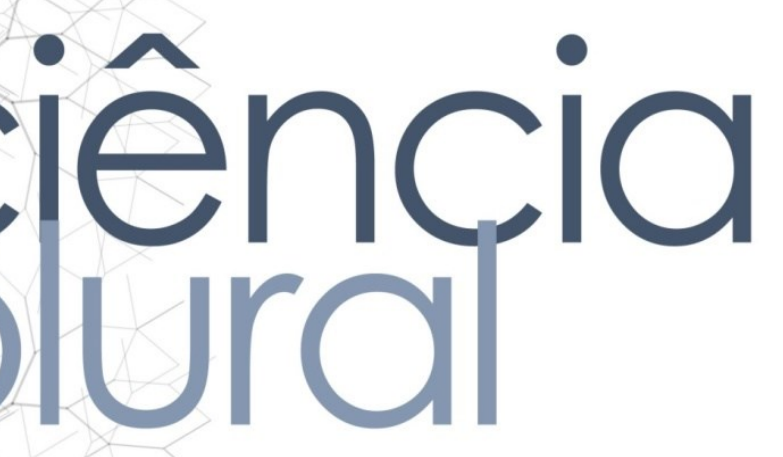

\title{
PERFIL DE PUBLICAÇÃO SOBRE NUTRIÇÃO ESPORTIVA NO APLICATIVO INSTAGRAM
}

\section{Profile of publication on sports nutrition on instagram app}

Jéssica Raissa Carlos Gomes • Graduando em Nutrição pela Faculdade de Ciências da Saúde do Trairi/UFRN. E-mail: jessica_rcg15@hotmail.com

Jarson Pedro da Costa Pereira • Graduando em Nutrição pela Faculdade de Ciências da Saúde do Trairi/UFRN. E-mail: jarsondelonge.oi@gmail.com

Tayse Priscila Pereira da Silva - Nutricionista graduada pela Faculdade de Ciências da Saúde do Trairi/UFRN. E-mail: tayse.priscila011@hotmail.com

Rayssa Gomes da Costa - Nutricionista da Prefeitura Municipal de Viçosa-RN. E-mail: nutriray@yahoo.com.br

Thaiz Mattos Sureira - Docente adjunta da Faculdade de Ciências da Saúde do Trairi/UFRN. E-mail: sureira78@gmail.com

Autora responsável pela correspondência:

Thaiz Mattos Sureira. E-mail: sureira78@gmail.com 


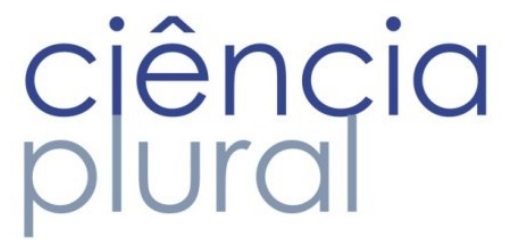

RESUMO

Introdução: A constante busca pela saúde e corpo perfeito, trouxe ao aplicativo Instagram um número considerável de usuários que utilizam o corpo como ferramenta discursiva e de convencimento, fazendo destes usuários guias de opinião diante dos demais. Objetivos: Conhecer o perfil das publicações sobre nutrição esportiva no Instagram. Metodologia: A amostra compreendeu 58 perfis dedicados ao tema nutrição esportiva, identificados após a pesquisa dos verbetes "Nutrição Esportiva" e "Nutrição Fitness", na área de busca do próprio aplicativo. Foi identificada a autoria e audiência dos perfis a partir dos dados apresentados em cada perfil avaliado, assim como, a quantificação dos seguidores e relacionou-se a audiência desses com as categorias analíticas de autoria. Resultados: Há cerca de 170 mil publicações sobre nutrição esportiva no Instagram. A maior parte foi publicada por perfis sem informações acerca da inserção profissional dos autores (Loja de suplementos - 53\%, sem definição - 17\%). Os nutricionistas formados e os especializados em nutrição esportiva foram uns dos que menos possuíam perfis no aplicativo (15\%), no entanto, foram os que apresentaram maior abrangência de público, provavelmente pelos comentários pertinentes e coerentes de quem conhece o que comenta. Conclusão: Faz-se necessário 0 envolvimento dos profissionais nutricionistas na veiculação desses conteúdos nas redes sociais, para que minimamente se tenha uma informação mais especializada, de mais credibilidade, de pessoas com formação na área em questão.

Palavras-chave: Meios de comunicação; Internet; Rede social; Nutrição; Esporte.

\section{ABSTRACT}

Introduction: The constant search for health and perfect body has brought to the mobile application Instagram a considerable number of users that use the body as a discursive and convincing tool, making these users opinion, guides to others. Objectives: Thus, we aimed to know the profile of sports nutrition publications on Instagram. Methodology: The sample included 58 profiles on sports nutrition, identified after the research of the terms "Sports Nutrition" and "Fitness Nutrition", in the search app area. The authorship and audience of the profiles were identified from the data presented in each profile evaluated, as well as the quantification of followers. The audience of these profiles was related to the analytical categories of authorship. Results: There are about 170 thousand publications about sports nutrition on Instagram. Most of these publications were published by profiles without information about the authors' professional insertion (Supplement shop - 53\%, without definition - 17\%). Nutritionists and those specialized in sports nutrition were among the ones that had less profiles in the app (15\%), however, they were the ones that presented greater public reach, probably by the pertinent and coherent comments of those who know what it says. Conclusion: It is necessary the involvement of nutritionists in the dissemination of these contents in social networks, so that there is at least a more specialized, more credible information of people with training in the area in question.

Key words: Media; Internet; Social network; Nutrition; Sport. 


\section{ciência plural}

\section{Introdução}

As mídias de comunicação como revistas, jornais, televisão e internet permitem que dados científicos sejam divulgados e tornem-se acessiveis à população geral (1), sendo assim, participam diretamente da difusão de conceitos e comportamentos relacionados aos hábitos e saúde (2). A divulgação dessas informações em saúde nos meios de comunicação é uma atitude útil para promover transformações no indivíduo em ser atuante e protagonista na sociedade a qual está inserido (3).

Hodiernamente, a sociedade vem sendo pautada em uma cultura que elege o corpo como padrão e fonte de identidade, estando atrelada a uma mídia que veicula publicidades que determina imagens de composição corporal ideais (4). Algumas dessas reportagens possuem a atividade fim de construir e/ou reconstruir uma nova prática social, como no que tange à melhora de aspectos relacionados ao processo de saúde-doença (5). Desta forma, a tecnologia vem realizando transformações no cotidiano das pessoas, por meio da influência constante que desenvolve sobre a forma de comunicação entre elas, bem como aspectos relacionados à alimentação, saúde e estética corporal (6).

As mídias sociais, por consequência do fácil acesso à conexão digital e a alta adesão de participação populacional a tais veículos virtuais de informação, delegam formatos e regras alimentares com elevado potencial de disseminação e influência, quando comparado aos padrões das mídias tradicionais, aumentando de maneira exacerbada o poder de reverberação de padrões na sociedade (7).

No Instagram, rede social cuja principal troca comunicativa é a das imagens, os atores sociais podem vincular seus conteúdos postados por meio de hashtags, que são um tipo de marcação, utilizada nas redes sociais e em outros meios, que serve para associar uma informação a um tópico ou discussão. Geralmente essas hashtags tornam-se links indexáveis pelos mecanismos de busca. As pessoas que se identificam com o perfil de um usuário do Instagram, usam as mesmas hashtags para se aproximarem, formando pequenas redes sociais dentro da comunidade maior, que é o próprio Instagram (8). Ao tratarse de analisar conteúdo relacionado com alimentos nas mídias sociais e ao analisar imagens compartilhadas no aplicativo Instagram, pesquisadores observaram uma proporção substancial de itens alimentares (9).

A constante busca pela saúde e corpo perfeito, trouxe a esse aplicativo um número considerável de usuários que utilizam o corpo como ferramenta discursiva e de convencimento, fazendo destes usuários guias de opinião diante dos demais. O poder de influência das redes sociais intercedendo comportamentos 
alimentares se mostra como discussão pertinente e urgente para os caminhos da nutrição esportiva, cabendo aos profissionais de nutrição voltados para essa área entender como esses conteúdos reverberam na população (10), haja vista, também, que o papel desses profissionais em meios de comunicação como esse, é divulgar informações científicas de forma adequada ao seu público (11).

Com base nas determinações supramencionadas, o presente estudo buscou conhecer o perfil das publicações no aplicativo Instagram sobre nutrição esportiva, quanto à audiência, número e autoria dos perfis destinados ao tema, assim como, seu público seguidor, através da identificação desses perfis e classificação dos mesmos, segundo os aspectos supracitados, no dia 27 do mês de julho de 2015.

\section{Método}

- Delineamento de estudo

Trata-se de um estudo descritivo, transversal, com abordagem quantitativa dos dados. Considerando a natureza das variáveis, os dados foram analisados descritivamente, por meio de frequências relativas e absolutas, a partir do software Microsoft Office Excel ${ }^{\circledR} 2010$.

\section{- Amostra e Amostragem}

A amostra do estudo foi composta por 58 perfis alocados no aplicativo Instagram dedicados ao tema nutrição esportiva, os quais foram identificados a partir de uma pesquisa na área de busca da própria rede social utilizando as palavras-chave: "Nutrição Esportiva" e "Nutrição Fitness". Foram excluídos os perfis com conteúdo privado ou sem conteúdo publicado. Para a análise dos dados foram utilizados métodos construídos pelos pesquisadores, compreendendo:

- Identificação dos autores e classificação dos perfis

A autoria das publicações foi identificada a partir dos dados de apresentação de cada perfil avaliado. As mesmas foram agrupadas nas seguintes categorias: Sem definição, Nutricionista, Loja de Suplementos Esportivos, Nutricionista Esportivo, Empresa de produção de refeições saudáveis e Outros, sendo distribuídos percentualmente, embasados no número total de perfis da amostra. 


\section{ciência plural}

- Audiência dos perfis

A audiência foi definida a partir da quantificação dos seguidores de cada perfil da amostra. Os mesmos foram agrupados nas categorias: audiência baixa (> $10 \leq 100$ seguidores), audiência mediana (>100 $\leq 500$ seguidores), audiência alta (> $500 \leq 2000$ seguidores) e audiência muito alta (> 2000 seguidores), segundo definição dos próprios autores do estudo. A distribuição percentual foi realizada com base no total de perfis avaliados. Já a relação percentual estabelecida entre as categorias de autoria das publicações dos perfis e as da audiência, foi realizada com base no total de perfis classificados em cada categoria analítica de autoria.

\section{Resultados e Discussão}

A pesquisa realizada dia 27 de julho de 2015, na área de busca do próprio aplicativo Instagram, utilizando as palavras-chave: "Nutrição Esportiva" e "Nutrição Fitness" retornou um total de aproximadamente 170 mil publicações, distribuídas em 58 perfis dedicados ao tema, e com conteúdo irrestrito quanto à privacidade.

Tabela 1: Distribuição percentual da autoria dos perfis sobre nutrição esportiva, alocados no aplicativo Instagram, em categorias analíticas, em relação ao número total de perfis avaliados. Natal-RN, 2015.

\begin{tabular}{lcc}
\hline Categoria profissional & $\mathbf{N}$ & $\%$ \\
\hline Outros & 3 & 5,2 \\
Estudante de nutrição & 2 & 3,5 \\
Loja de suplementos & 31 & 53,0 \\
Nutricionista & 3 & 5,2 \\
Nutricionista esportivo & 6 & 10,3 \\
Sem definição & 10 & 17,0 \\
Empresa de produção de refeições & 3 & 5,2 \\
saudáveis & & \\
Total & 58 & 100,0 \\
\hline
\end{tabular}


- Identificação dos autores e classificação dos perfis

A maior parte do conteúdo sobre nutrição esportiva publicado no aplicativo Instagram advém de perfis sem informações acerca da inserção profissional dos mesmos. Os melhores capacitados para realização da educação nutricional e alimentar através de mídias sociais, os nutricionistas formados e os especializados em nutrição esportiva, foram uns dos que menos possuíam perfis no aplicativo (Tabela 1). Isso abre precedentes e poderá representar um perigo no que se refere à veiculação de informações equivocadas para um público leigo, o qual poderá ser negativamente influenciado, chegando inclusive a desenvolver transtornos de ordem alimentar.

\section{- Audiência dos perfis}

Os perfis das redes sociais dedicados ao tema, normalmente, desenvolvem um discurso que reforça a ideia de perfeição física, conseguida por meio de esforço nos exercícios e na alimentação correta, explorando uma obsessão corporal e alimentar de cunho semelhante ao religioso, tendo em vista que estimula o culto ao corpo. Esse discurso é potencializado graças às redes sociais de compartilhamento de informações, especialmente o Instagram, onde os adeptos do universo fitness divulgam o seu "estilo de vida fitness" (3), os quais acabam influenciando o hábito alimentar de outros usuários desse aplicativo, pois como relatou Azevedo (8), esses hábitos são definidos cada vez mais por parâmetros científicos e determinados pela indústria do marketing. Por isso, torna-se evidente a necessidade do profissional nutricionista se envolver mais nesse meio, que tragam comentários baseados em evidências e conhecimento científico, baseados em pesquisas e não apenas em observações empíricas.

Quanto à audiência dos perfis direcionados ao tema nutrição esportiva, no Instagram, observou-se que $75 \%$ do conteúdo veiculado possui audiência alta e muito alta, chegando a atingir um quantitativo superior a 180 mil indivíduos seguidores (Tabela 2). Os perfis da amostra gerenciados por nutricionistas esportivos foram os que apresentaram maior abrangência de público, seguido daqueles gerenciados por estabelecimentos distribuidores de refeições "fit" ou de suplementos esportivos (categoria "outros"), cujas lojas responsáveis pela venda dos mesmos, apresentaram perfis, nessa mídia, com uma audiência compreendida entre as categorias de audiência alta e muito alta. 
Tabela 2: Distribuição percentual da audiência dos perfis, sobre nutrição esportiva, alocados no aplicativo Instagram, em categorias analíticas, considerando o número total de perfis avaliados. Natal-RN, 2015

\begin{tabular}{lcc}
\hline \multicolumn{1}{c}{ Audiência } & $\mathbf{n}$ & \% \\
\hline Audiência baixa (> $10 \leq 100$ seguidores) & 4 & 6,9 \\
$\begin{array}{l}\text { Audiência mediana (>100 } \leq \mathbf{5 0 0} \\
\text { seguidores) }\end{array}$ & 10 & 17,0 \\
Audiência alta (> $500 \leq \mathbf{2 0 0 0}$ seguidores) & 19 & 33,0 \\
Audiência muito alta (> 2000 seguidores) & 25 & 43,0 \\
TOTAL & 58 & 100 \\
\hline
\end{tabular}

Importante ressaltar, também, o expressivo percentual (30\%) de audiência acima de 2000 mil seguidores, de perfis sem autoria definida (Gráfico 1). Esses resultados demonstram a divergência que há na ocupação do espaço de influência nos meios de comunicação de massa digitais. Os profissionais de saúde têm opiniões diversas em relação à internet, de um lado, os adeptos sem restrições e também, em volume um pouco menor, os entendedores do poder da internet como ambiente de viralização da imagem e autopromoção, conduta, no caso dos nutricionistas, contrária ao que é exigido no código de ética da profissão, Capítulo XII da Publicidade. Art. 22 (9). Do outro lado, aqueles que se posicionam totalmente contra o uso dessas mídias e a dependência causada pelas mesmas (10). Esse tipo de comportamento abre precedentes para a ocupação crescente desse lugar por outros profissionais não capacitados para tal ou até mesmo leigos (blogueiros, comerciantes, etc.) como podemos observar nesse estudo. 


\section{ciência \\ plural}

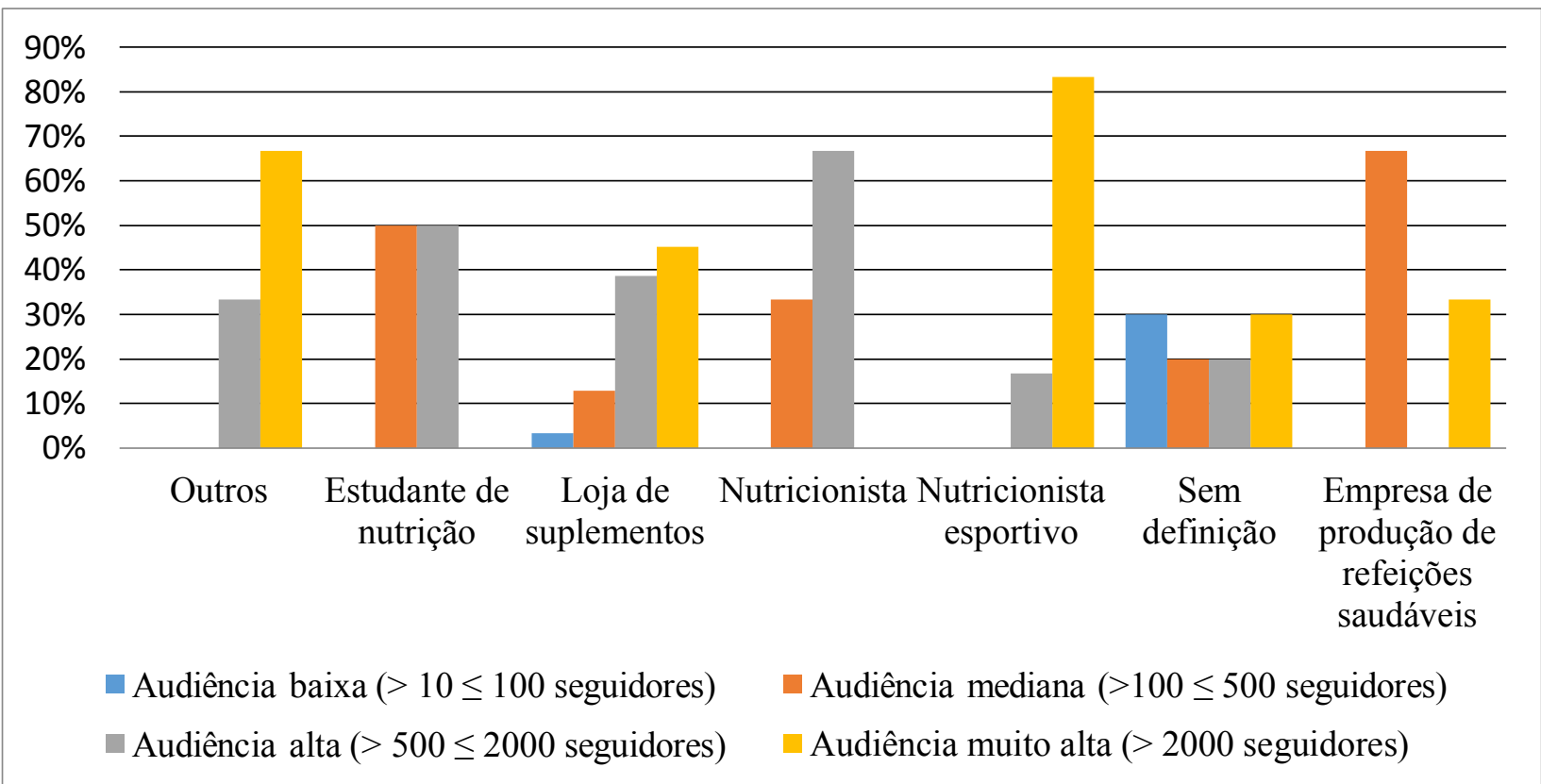

Gráfico 1: Distribuição percentual da audiência dos perfis sobre nutrição esportiva, alocados no aplicativo Instagram, categorizados segundo a autoria de suas publicações, considerando o número total de perfis por categoria. Natal-RN, 2015.

\section{Conclusão}

No ato da realização da pesquisa havia no aplicativo Instagram um total de 170 mil publicações sobre o tema nutrição esportiva, distribuídas em 58 perfis de conteúdo irrestrito quanto à privacidade. Os mesmos, em sua maioria, não apresentavam definição de inserção profissional dos autores das publicações, sendo as lojas de suplementos as principais responsáveis pelo conteúdo de nutrição esportiva publicado no aplicativo. Em contrapartida, os perfis de nutricionistas esportivos foram os que apresentaram maior abrangência de público, comportamento semelhante em um terço dos perfis sem autoria definida. Portanto, torna-se necessário o envolvimento dos profissionais nutricionistas na veiculação desses conteúdos nas redes sociais, cabendo também a estes a divulgação de conhecimento científico para o público, além de produzir novos estudos no intuito de avaliar o teor das informações veiculadas, baseadas em evidências e conhecimento científico gerado por profissionais da área.

\section{Referências}

1. Pechula MR. A ciência nos meios de comunicação de massa: divulgação de conhecimento ou reforço do imaginário social? Ciênc. educ. (Bauru) 2007;13(2):211-22. 


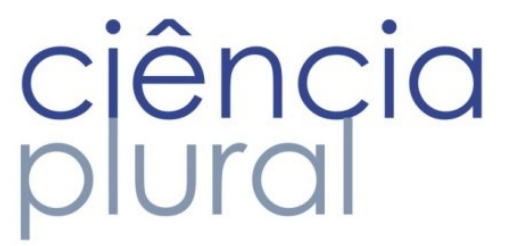

2. Soares MC, Milanez IAP. A temática de saúde nos meios massivos. 2002. [acesso em 10 abril 2017]. Disponível em: http://encipecom.metodista.br/mediawiki/im ages/5/52/08__TEMaTICA_DE_SAuDE_NOS_MEIOS_MASSIVOS.pdf

3. Luz LH. A pílula da longevidade à venda nas páginas da Revista Veja. Intercom - Revista Brasileira de Ciências da Comunicação. 2010;33(1):257-76.

4. Martins, DF et al., Satisfação com a imagem corporal e auto conceito em adolescentes. Psicologia: Teoria e Prática. 2008; 10(2): 94-105.

5. Teixeira RA, Min LL, Toledo VR. A divulgação do AVC por dois meios de comunicação de massa. Revista Eletrônica de Jornalismo Científico 10 Abr 2017. [acessado em 17 abril 2012]. Disponível em: http://www.comciencia.br/comciencia/?secti on=8\&edicao=47\&id=588

6. Othon,R.; Coelho, M. A influência do self reality show online na apropriação de práticas de alimentação saudável no Instagram. Razón Y Palabra. 2016; 20(3): 425-442

7. Jacob H. Redes sociais, mulheres e corpo: um estudo da linguagem fitness na rede social Instagram. RevCommunicar. 2014; 14(1): 88-105.

8. Batista G, Rodrigues RA Construção de Identidade na "Geração Fitness" do Instagram: a representação do eu e do corpo no ciberespaço[internet] [acessado em: 10 jul 2015].Disponívelem:http://www. intercom.org.br/sis/2014/resumos/R9-1378-1.pdf

9. Holmberg $\mathrm{C}$ et al. Adolescents' presentationoffood in social media: Anexplorativestudy. Appetite. 2016; $121-129$.

10. Freire C. Alimentação na mídia impressa: uma análise de conteúdo da Revista Boa Forma. [internet]. 2011. [acesso em 2016 out 20]. Disponível em: http://www.lume.ufrgs.br/handle/10183/34061. 11. American Dietetic Association (ADA). In Dramatically Increasing Numbers, TV and Magazines Remain Consumers' Most Popular Sources of Nutrition Information, American Dietetic Association Survey Shows. [acessado em 2016 out 20]. Disponível em: http://www.eatright.org.

12. Azevedo E. Riscos e controvérsias na construção social do conceito de alimento saudável: 0 caso da soja. Rev Saúde Pública. 2011; 45(4):781-788.

13. Brasil. Conselho Federal De Nutricionistas (CFN). Código de Ética. Resolução CFN 334/2004[internet][acessado em: 20 out 2016]. Brasília: CFN. 2004. Disponível em: http://www.cfn.org.br/novosite/pdf/codigo/codigo\%20de\%20etica_nova\%20redacao.pdf

14. Faria JT. Uso de Mídias Sociais no Marketing em Saúde. RevMovimenta. 2014; 7(4): 795-796. 